\title{
PRIMEIRA ETAPA DE RECONHECIMENTO ARQUEOLÓGICO DO MÉDIO/BAIXO VALE DO RIO PIRAPÓ, PARANÁ*
}

A região noroeste do Estado do Paraná possui imenso potencial arqueológico, segundo informação de vários projetos de levantamento já realizados e das fontes coloniais (Noelli, no prelo 1). As evidências humanas mais antigas conhecidas até agora são datadas em 8 mil A.P. (Chmyz 1992), vinculadas a um amplo horizonte cultural relacionado à Tradição Umbu, que perdurou até cerca de 2 mil A.P., quando a região começou a ser colonizada por populações Guarani provenientes das bacias dos rios Paraguai e Paraná (Brochado 1984; Noelli 2000 a, b). Os Guarani do Guairá, antiga denominação colonial da região, conforme as projeções de Melià (1988), poderiam alcançar até 1 milhão de pessoas no início do século XVII, aproximadamente 1/4 da população atual (Censo-IBGE 2000).

Contribuindo para as pesquisas no noroeste paranaense, desde 1996 realizamos trabalhos de reconhecimento limitados ao registro de ocorrências de superfície, sem qualquer forma de intervenção arqueológica. Nossa meta é escolher uma área de pesquisa permanente, onde desenvolveremos atividades de arqueologia científica e pública de longa duração em nível regional (Noelli, Silva e Mota 2000). Realizamos levantamentos sistemáticos no rio Tibagi, municípios de Londrina, Tamarana e São Jerônimo da Serra (1996-1997); rio Paraná, municípios de Guaíra, Altônia, Vila Alta (19961999); na APA Federal do Noroeste do Paraná, municípios de Santa Cruz do Monte Castelo, Querência do Norte, Porto Rico, São Pedro do Paraná, Marilena, Nova Londrina e Diamante do Norte (2000); no rio Pirapó, municípios de Lobato, Colorado, Cruzeiro do Sul, Uniflor, Paranacity, Atalaia e Flórida (2001); além de registrar sítios arqueológicos isolados em outros municípios (1994-2002), atendendo notificações feitas à Universidade Estadual de Maringá. Também realizamos um trabalho de conscientização sobre a

(*) Convênio Prefeitura de Lobato e Universidade Estadual de Maringá, financiado pelo Fundo Nacional do Meio Ambiente (Projeto 012/2000 - CPC, Processo Plano de Manejo das RPPN de Lobato). necessidade de preservar os sítios e evidências materiais e disseminação de conhecimento arqueológico junto às populações das Vilas Rurais do vale do rio Ivaí (2001-2002). Assim, localizamos um total de 180 sítios, além de várias ocorrências isoladas e peças doadas (Noelli 1998, m.s. 1; Noelli et al., no prelo 2 e 3; Mota, Noelli e Silva 1996; Silva e Noelli 1996).

Esta nota de pesquisa divulga os resultados de campo obtidos no baixo/médio vale do rio Pirapó, entre julho e dezembro de 2000. Trata-se da primeira de duas etapas de reconhecimento de todo o município de Lobato, que foi dividido em faixas de survey: 1) perímetro do município; 2) interior do município. Investigamos todo o perímetro, numa faixa média de $1 \mathrm{~km}$ de largura, subdividida em linhas para orientar caminhamentos sistemáticos. Também percorremos trechos fora do perímetro de Lobato, ao longo dos rios Pirapó e Bandeirantes, nos municípios de Colorado, Paranacity, Cruzeiro do Sul, Uniflor, Atalaia e Flórida. Percorremos porções do interior de Lobato, subindo alguns cursos d'água até as nascentes. As demais equipes do convênio Prefeitura de Lobato-ITCA/UEM fizeram completos inventários geológicos, pedológicos, hídricos, botânicos e zoológicos, que serão incorporados e analisados na continuidade da pesquisa arqueológica. Como não realizamos nenhuma modalidade de escavação ou de coleta de evidências, ainda não dispomos de dados que permitam especificar a área efetiva e a estratigrafia dos sítios localizados. A realização destas e de outras atividades arqueológicas iniciarão em 2004, após obtermos autorização do IPHAN e dispormos dos recursos, previstos pelo ITCA/UEM.

Localizamos 44 sítios e 3 ocorrências cerâmicas isoladas até o presente (Fig. 1). A observação in situ permitiu classificar as evidências cerâmicas como pertencentes à Tradição Guarani, enquanto os sítios que apresentaram apenas evidências líticas não foram classificados, fato que ocorrerá na continuidade das pesquisas. As evidências cerâmicas estão em 39 sítios e 3 ocorrências isoladas, enquanto os sítios apenas com evidências líticas somam 5 unidades. Levantamos as referências mais 
elementares para identificar os sítios, identificando apenas o município, a localidade, a altitude, a distância da água mais próxima e o compartimento topográfico, bem como batizamos os sítios com nomes e códigos (Tabela 1). A maioria dos sítios apresentou mais de 500 fragmentos por concentração, sendo que alguns possuem várias concentrações e milhares de fragmentos sobre a superfície dos terrenos perturbados superficialmente pelo plantio de cana de açúcar.

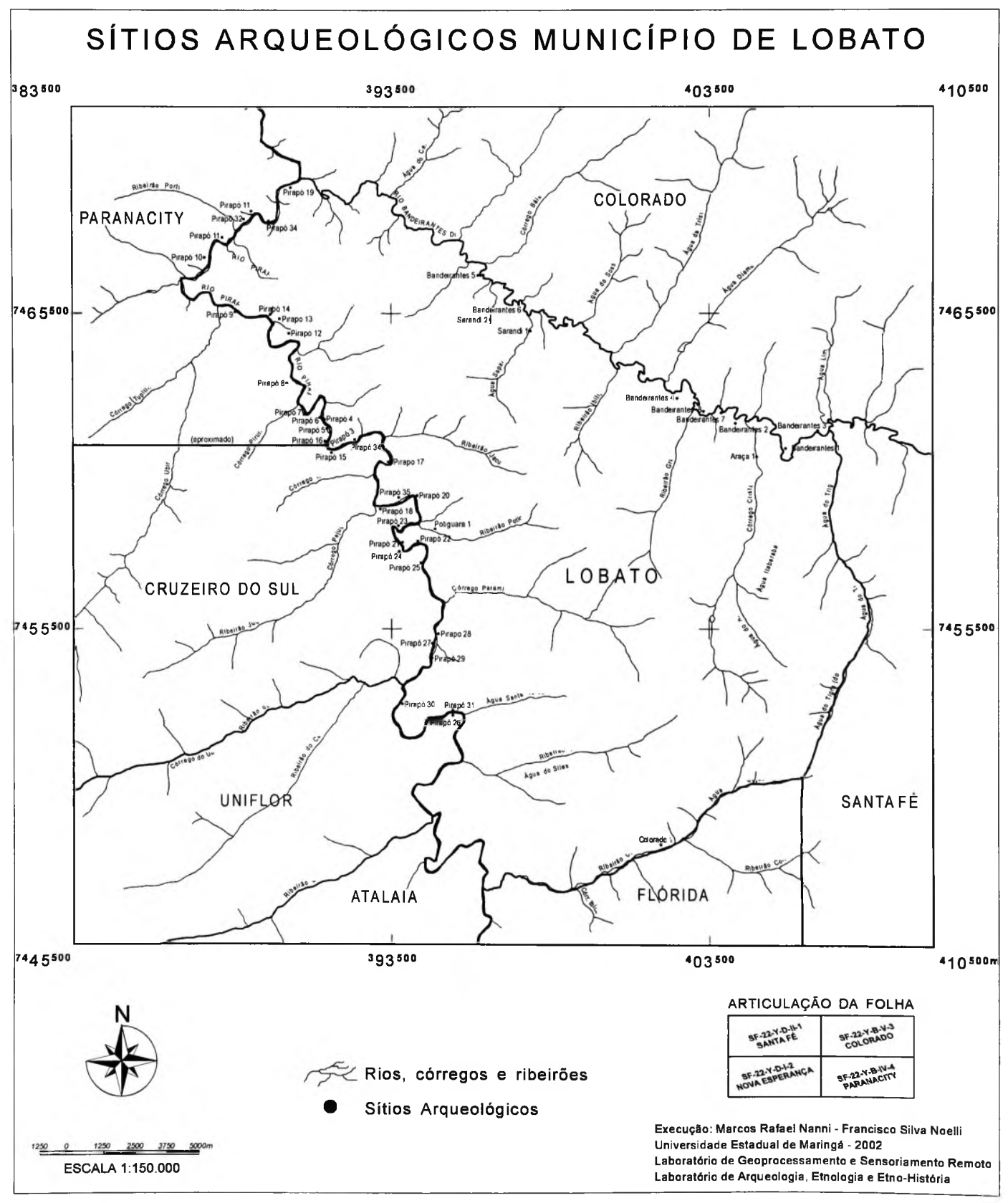


TABELA 1

Sítios e ocorrências arqueológicas

\begin{tabular}{|c|c|c|c|c|c|c|c|c|}
\hline \multirow{2}{*}{$\begin{array}{l}\text { Município } \\
\text { Colorado }\end{array}$} & \multirow{2}{*}{$\begin{array}{c}\text { Nome do Sítio } \\
\text { Bandeirantes 1 }\end{array}$} & \multirow{2}{*}{$\begin{array}{c}\text { Código } \\
\text { PR - BD-01 }\end{array}$} & \multirow{2}{*}{$\begin{array}{c}\text { Categoria } \\
\text { Guarani }\end{array}$} & \multicolumn{2}{|c|}{$\begin{array}{c}\text { Coordenadas } \\
\text { UTM }\end{array}$} & \multirow{2}{*}{$\begin{array}{c}\begin{array}{c}\text { Altitude } \\
\text { (m) }\end{array} \\
344\end{array}$} & \multirow{2}{*}{$\begin{array}{c}\begin{array}{c}\text { Água + } \\
\text { próxima } \\
\text { (m) }\end{array} \\
50\end{array}$} & \multirow{2}{*}{$\begin{array}{c}\begin{array}{c}\text { Compartimento } \\
\text { Topográfico }\end{array} \\
\text { Encosta }\end{array}$} \\
\hline & & & & 7461219 & 405897 & & & \\
\hline Colorado & Bandeirantes 3 & PR - BD - 03 & Lítico & & & & 30 & Encosta \\
\hline Colorado & Bandeirantes 8 & PR - BD - 08 & Guarani & 7462401 & 403330 & 361 & 10 & Encosta \\
\hline Cruzeiro do Sul & Pirapó 15 & PR - CZ-01 & Guarani & 7461089 & 391620 & 346 & 18 & Encosta \\
\hline Cruzeiro do Sul & Pirapó 17 & $\mathrm{PR}-\mathrm{CZ}-02$ & Guarani & 7460779 & 393435 & 342 & 12 & Encosta \\
\hline Cruzeiro do Sul & Pirapó 18 & PR - CZ -03 & Lítico & 7459298 & 393151 & 354 & 2 & Encosta \\
\hline Cruzeiro do Sul & Pirapó 23 & PR - CZ - 04 & Lítico & 7458774 & 393718 & 351 & 10 & Terraço \\
\hline Cruzeiro do Sul & Pirapó 24 & PR - CZ - 06 & $\mathrm{OCI}^{1}$ & 7457955 & 393744 & 357 & 35 & Encosta \\
\hline Cruzeiro do Sul & Pirapó 25 & PR - CZ- 07 & OCI & 7457588 & 394422 & 368 & 30 & Encosta \\
\hline Cruzeiro do Sul & Pirapó 27 & PR - CZ- 05 & Lítico & 7455040 & 394782 & 333 & 25 & Encosta \\
\hline Lobato & Araçá 1 & PR - LB - 22 & Guarani & 7460953 & 404998 & 356 & 12 & Encosta \\
\hline Lobato & Bandeirantes 2 & PR - LB - 01 & Guarani & 7462012 & 404331 & 345 & 42 & Encosta \\
\hline Lobato & Bandeirantes 4 & PR - LB - 02 & Guarani & 7462810 & 402496 & 362 & 20 & Encosta \\
\hline Lobato & Bandeirantes 5 & PR - LB - 05 & Guarani & 7466701 & 396237 & 334 & 18 & Encosta \\
\hline Lobato & Bandeirantes 6 & PR - LB - 19 & Guarani & & & & 18 & Encosta \\
\hline Lobato & Bandeirantes 7 & PR - LB - 20 & Guarani & 7462351 & 403884 & 368 & 15 & Encosta \\
\hline Lobato & Colorado 1 & PR - LB - 23 & Guarani & 7448675 & 401970 & 366 & 15 & Encosta \\
\hline Lobato & Pirapó 12 & PR - LB - 09 & Guarani & 7464868 & 390286 & 351 & 32 & Encosta \\
\hline Lobato & Pirapó 13 & PR - LB - 10 & Guarani & 7465329 & 389991 & 347 & 20 & Encosta \\
\hline Lobato & Pirapó 14 & PR - LB - 11 & Guarani & 7465475 & 389709 & 356 & 30 & Encosta \\
\hline Lobato & Pirapó 19 & PR - LB - 12 & Guarani & 7469450 & 390353 & 346 & 18 & Encosta \\
\hline Lobato & Pirapó 20 & PR - LB - 13 & Guarani & 7459721 & 394306 & 342 & 15 & Encosta \\
\hline Lobato & Pirapó 21 & PR - LB - 14 & Guarani & 7458203 & 393822 & 351 & 15 & Topo \\
\hline Lobato & Pirapó 22 & PR - LB - 15 & Guarani & 7458280 & 394329 & 350 & 15 & Topo \\
\hline Lobato & Pirapó 28 & PR - LB - 16 & Guarani & 7455344 & 394972 & 376 & 18 & Topo \\
\hline Lobato & Pirapó 29 & PR - LB - 25 & $\mathrm{OCI}$ & 7454574 & 394768 & 373 & 2 & Encosta \\
\hline Lobato & Pirapó 3 & PR - LB - 07 & Guarani & 7461376 & 391521 & 338 & 32 & Encosta \\
\hline Lobato & Pirapó 30 & PR - LB - 17 & Guarani & 7453119 & 393832 & 373 & 12 & Encosta \\
\hline Lobato & Pirapó 32 & PR - LB - 18 & Guarani & 7468471 & 388888 & 337 & 30 & Encosta \\
\hline Lobato & Pirapó 35 & PR - LB - 06 & Guarani & 7459669 & 393726 & 326 & 15 & Encosta \\
\hline Lobato & Pirapó 36 & PR - LB - 24 & Lítico & 7461474 & 392432 & 341 & 35 & Encosta \\
\hline Lobato & Pirapó 4 & PR - LB - 08 & Guarani & 7462144 & 391402 & 328 & 33 & Encosta \\
\hline Lobato & Potiguara 1 & PR - LB - 21 & Guarani & 7458662 & 394870 & 362 & 30 & Encosta \\
\hline Lobato & Sarandi 1 & PR - LB - 03 & Guarani & 7464943 & 397881 & 338 & 5 & Topo \\
\hline Lobato & Sarandi 2 & PR - LB - 04 & Guarani & 7465307 & 396631 & 351 & 10 & Encosta \\
\hline Paranacity & Pirapó 10 & PR - PT - 06 & Guarani & 7467269 & 387650 & 327 & 20 & Encosta \\
\hline Paranacity & Pirapó 11 & PR - PT - 07 & Guarani & 7467895 & 388211 & 348 & 18 & Encosta \\
\hline Paranacity & Pirapó 16 & PR - PT - 12 & Guarani & 7461449 & 391431 & 350 & 15 & Encosta \\
\hline Paranacity & Pirapó 31 & PR - PT - 08 & Guarani & 7452894 & 395430 & 361 & 22 & Encosta \\
\hline Paranacity & Pirapó 33 & PR - PT - 09 & Guarani & 7468708 & 389128 & 350 & 30 & Encosta \\
\hline Paranacity & Pirapó 34 & PR - PT 10 & Guarani & 7468414 & 389660 & 344 & 20 & Encosta \\
\hline Paranacity & Pirapó 5 & PR - PT - 01 & Guarani & 7461853 & 391469 & 327 & 20 & Encosta \\
\hline Paranacity & Pirapó 6 & PR - PT - 02 & Guarani & 7462206 & 391322 & 329 & 18 & Encosta \\
\hline Paranacity & Pirapó 7 & PR - PT - 03 & Guarani & 7462294 & 390796 & 352 & 15 & Encosta \\
\hline Paranacity & Pirapó 8 & PR - PT - 04 & Guarani & 7463296 & 390216 & 358 & 200 & Encosta \\
\hline Paranacity & Pirapó 9 & PR - PT - 05 & Guarani & 7465551 & 388609 & 348 & 33 & Encosta \\
\hline Uniflor & Pirapó 26 & PR - UN - 01 & Guarani & 7452762 & 395422 & 367 & 40 & Encosta \\
\hline
\end{tabular}

(1)OCI = Ocorrência cerâmica isolada 


\section{Referências bibliográficas}

\section{BROCHADO, J.P.}

1984 An ecological model of the spread of pottery and agriculture into Eastern South America. Urbana-Champaign, University of Illinois at Urbana-Champaign, 1984. Tese (Doutorado).

CHMYZ, I.

1992 Projeto Arqueológico Rosana-Taquaruçu. Curitiba: UFPR-CESP.

MELIÀ, B.

1988 El Guarani conquistado y reducido. Asunción: CEADUC

MOTA, L.T.; NOELLI, F.S.; SILVA, F.A.

1996 Pãri: armadilha de pesca utilizada pelos índios Kaingang no sul do Brasil. Universidade e Sociedade, Maringá, 16:21-25.

\section{NOELLI, F.S.}

1998 Nota sobre a presença da Tradição Umbu no médio-baixo Ivaí, Paraná. Revista do CEPA, Santa Cruz do Sul, 22(27):101-105.

2000a A ocupação humana na região sul do Brasil: Arqueologia, debates e perspectivas -1872 2000. Revista USP, São Paulo, 44: 218-269.

$2000 \mathrm{~b}$ A Presença Guarani desde 2.000 anos atrás: contribuição para a História da ocupação humana do Paraná. In: R.C. Rolim; S.A. Pellegrini e R.B. Dias (Orgs.) História, espaço e meio ambiente (VI Encontro Regional de História, ANPUH $P R$ ). Maringá, ANPUH: 403-414.

prelo 1 Catálogo de sítios arqueológicos de populações ceramistas do sul do Brasil, Uruguai, Paraguai oriental e Argentina (Províncias de Misiones, Corrientes, Entre Ríos e Buenos Aires). Maringá: Laboratório de Arqueologia, Etnologia e Etno-História/Programa Interdisciplinar de Estudos de Populações. m.s.1 Registro de sítios arqueológicos do Laboratório de Arqueologia, Etnologia e Etno-História/Programa Interdisciplinar de Estudos de Populações. ${ }^{1}$

NOELLI, F.S.; MOTA, L.T.; NANNI, M.R.; LAVADO, M.C.; OLIVEIRA, E.R.; PANEK, Jr., C.A.; SIMÃO, A.P.; MARQUES, A.J.; SILVA, J.B.; CASTILHO, W.

prelo 2 Agricultores Guarani na pré-história da região noroeste do Paraná. Universidade e Sociedade, Maringá, 18.

NOELLI, F.S.; MOTA, L.T.; NANNI, M.R.; LAVADO, M.C.; OLIVEIRA, E.R.; PANEK, Jr., C.A.; SIMÃO, A.P.; SILVA, J.B.; CASTILHO, W.

prelo 3 Pesquisas arqueológicas na região noroeste do Paraná, entre os rios Paranapanema e Ivaí. Curitiba: Anais do $8^{\circ}$ Encontro da ANPUH-PR (2002).

NOELLI, F.S.; MOTA, L.T.; SILVA, F.A.

1996 Pari: armadilha de pesca no sul do Brasil e a arqueologia. Coleção Arqueologia, Porto Alegre, 1(2): 435-446. (Anais da VIII Reunião Científica da Sociedade Arqueologia Brasileira).

NOELLI, F.S.; SILVA, F.A.; MOTA, L.T.

2000 Projeto de pesquisa arqueológica no noroeste do Paraná (1996-1997). CD-ROM. S.M. de Souza (Org.). Anais da IX Reunião Científica da Sociedade Arqueologia Brasileira. Rio de Janeiro.

SILVA, F.A.; NOELLI, F.S.

1996 A ocupação do espaço na Terra Indígena Apucarana: elementos para uma reflexão interdisciplinar. Revista do CEPA, Santa Cruz do Sul, 20 (24):27-36.

Francisco Silva Noelli** Lúcio Tadeu Mota**

Marcos Rafael Nanni*** Margarida Cardozo Lavado****

Carlos Panek Jr** Eurides Roque de Oliveira** Ana Paula Simão** Eder Novak** Washington C. Castilho**

(1) Texto em fase de elaboração, a ser publicado pela revista Geonotas, Maringá.

(**) Laboratório de Arqueologia, Etnologia e Etno-História/Universidade Estadual de Maringá.

(***) Departamento de Agronomia/Universidade Estadual de Maringá.

(****) Mestrado em Arqueologia/MAE - USP. 